TRANSACTIONS OF THE

AMERICAN MATHEMATICAL SOCIETY

Volume 357, Number 6, Pages 2467-2481

S 0002-9947(04)03588-3

Article electronically published on November 4, 2004

\title{
SMOOTHNESS OF EQUISINGULAR FAMILIES OF CURVES
}

\author{
THOMAS KEILEN
}

\begin{abstract}
Francesco Severi (1921) showed that equisingular families of plane nodal curves are T-smooth, i.e. smooth of the expected dimension, whenever they are non-empty. For families with more complicated singularities this is no longer true. Given a divisor $D$ on a smooth projective surface $\Sigma$ it thus makes sense to look for conditions which ensure that the family $V_{|D|}^{i r r}\left(\mathcal{S}_{1}, \ldots, \mathcal{S}_{r}\right)$ of irreducible curves in the linear system $|D|_{l}$ with precisely $r$ singular points of types $\mathcal{S}_{1}, \ldots, \mathcal{S}_{r}$ is T-smooth. Considering different surfaces including the projective plane, general surfaces in $\mathbb{P}_{\mathbb{C}}^{3}$, products of curves and geometrically ruled surfaces, we produce a sufficient condition of the type

$$
\sum_{i=1}^{r} \gamma_{\alpha}\left(\mathcal{S}_{i}\right)<\gamma \cdot\left(D-K_{\Sigma}\right)^{2}
$$

where $\gamma_{\alpha}$ is some invariant of the singularity type and $\gamma$ is some constant. This generalises the results of Greuel, Lossen, and Shustin (2001) for the plane case, combining their methods and the method of Bogomolov instability. For many singularity types the $\gamma_{\alpha}$-invariant leads to essentially better conditions than the invariants used by Greuel, Lossen, and Shustin (1997), and for most classes of geometrically ruled surfaces our results are the first known for T-smoothness at all.
\end{abstract}

\section{INTRODUCTION}

The varieties $V_{|D|}\left(r A_{1}\right)$ (respectively the open subvarieties $V_{|D|}^{i r r}\left(r A_{1}\right)$ ) of reduced (respectively reduced and irreducible) nodal curves in a fixed linear system $|D|_{l}$ on a smooth projective surface $\Sigma$ are also called Severi varieties. When $\Sigma=\mathbb{P}_{\mathbb{C}}^{2}$ Severi showed that these varieties are smooth of the expected dimension whenever they are non-empty - that is, nodes always impose independent conditions. It seems natural to study this question on other surfaces, but it is not surprising that the situation becomes harder.

Tannenbaum showed in Tan82] that on K3-surfaces $V_{|D|}\left(r A_{1}\right)$ is also always smooth, but that, however, the dimension is larger than the expected one and thus $V_{|D|}\left(r A_{1}\right)$ is not T-smooth in this situation. If we restrict our attention to the subvariety $V_{|D|}^{i r r}\left(r A_{1}\right)$ of irreducible curves with $r$ nodes, then we gain T-smoothness

Received by the editors September 1, 2003 and, in revised form, December 12, 2003.

2000 Mathematics Subject Classification. Primary 14H10, 14H15, 14H20; Secondary 14J26, $14 \mathrm{~J} 27,14 \mathrm{~J} 28,14 \mathrm{~J} 70$.

Key words and phrases. Algebraic geometry, singularity theory.

The author was partially supported by the German Israeli Foundation for Research and Development, by the Hermann Minkowski - Minerva Center for Geometry at Tel Aviv University and by EAGER. 
again whenever the variety is non-empty. That is, while on a K3-surface the conditions which nodes impose on irreducible curves are always independent, they impose dependent conditions on reducible curves.

On more complicated surfaces the situation becomes even worse. Chiantini and Sernesi study in [CS97] Severi varieties on surfaces in $\mathbb{P}_{\mathbb{C}}^{3}$. They show that on a generic quintic $\Sigma$ in $\mathbb{P}_{\mathbb{C}}^{3}$ with hyperplane section $H$ the variety $V_{|d H|}^{i r r}\left(\frac{5 d(d-2)}{4} \cdot A_{1}\right)$ has a non-smooth reduced component of the expected dimension, if $d$ is even. They construct their examples by intersecting a general cone over $\Sigma$ in $\mathbb{P}_{\mathbb{C}}^{4}$ with a general complete intersection surface of type $\left(2, \frac{d}{2}\right)$ in $\mathbb{P}_{\mathbb{C}}^{4}$ and projecting the resulting curve to $\Sigma$ in $\mathbb{P}_{\mathbb{C}}^{3}$. Moreover, Chiantini and Ciliberto give in CC99 examples showing that the Severi varieties $V_{|d H|}^{i r r}\left(r A_{1}\right)$ on a surface in $\mathbb{P}_{\mathbb{C}}^{3}$ may also have components of dimension larger than the expected one.

Hence, one can only ask for numerical conditions ensuring that $V_{|d H|}^{i r r}\left(r A_{1}\right)$ is T-smooth, and Chiantini and Sernesi answer this question by showing that on a surface of degree $n \geq 5$ the condition

$$
r<\frac{d(d-2 n+8) n}{4}
$$

implies that $V_{|d H|}^{i r r}\left(r A_{1}\right)$ is T-smooth for $d>2 n-8$. Note that the above example shows that this bound is even sharp. Actually Chiantini and Sernesi prove a somewhat more general result for surfaces with ample canonical divisor $K_{\Sigma}$ and curves which are in $\left|p K_{\Sigma}\right|_{l}$ for some $p \in \mathbb{Q}$. For their proof they suppose that for some curve $C \in V_{|d H|}^{i r r}\left(r A_{1}\right)$ the cohomology group $H^{1}\left(\Sigma, \mathcal{J}_{X^{*}(C) / \Sigma}(D)\right)$ does not vanish and derive from this the existence of a Bogomolov unstable rank-two bundle $E$. This bundle in turn provides them with a curve $\Delta$ of small degree realising a large part of the zero-dimensional scheme $X^{*}(C)$, which leads to the desired contradiction.

This is basically the same approach used in GLS97. However, they allow arbitrary singularities rather than only nodes, and get in the case of a surface in $\mathbb{P}_{\mathbb{C}}^{3}$ of degree $n$

$$
\sum_{i=1}^{r}\left(\tau_{c i}^{*}\left(\mathcal{S}_{i}\right)+1\right)^{2}<d \cdot\left(d-(n-4) \cdot \max \left\{\tau_{c i}^{*}\left(\mathcal{S}_{i}\right)+1 \mid i=1, \ldots, r\right\}\right) \cdot n
$$

as a main condition for T-smoothness of $V_{|d H|}^{i r r}\left(\mathcal{S}_{1}, \ldots, \mathcal{S}_{r}\right)$, which for nodal curves coincides with (1.1). Moreover, for families of plane curves of degree $d$ their result gives

$$
\sum_{i=1}^{r}\left(\tau_{c i}^{*}\left(\mathcal{S}_{i}\right)+1\right)^{2}<d^{2}+6 d
$$

as a sufficient condition for T-smoothness, which is weaker than the sufficient condition

$$
\sum_{i=1}^{r} \gamma_{1}^{*}\left(\mathcal{S}_{i}\right) \leq(d+3)^{2}
$$

derived in [GLS00] and GLS01] using the Castelnuovo function in order to provide a curve of small degree which realises a large part of $X^{*}(C)$. The advantage of the $\gamma_{1}^{*}$-invariant is that, while always bounded from above by $\left(\tau_{c i}^{*}+1\right)^{2}$, in many cases 
it is substantially smaller - e.g. for an ordinary $m$-fold point $M_{m}, m \geq 3$, we have $\gamma_{1}^{e s}\left(M_{m}\right)=2 m^{2}$, while

$$
\left(\tau_{c i}^{e s}\left(M_{m}\right)+1\right)^{2} \geq \frac{\left(m^{2}+2 m+4\right)^{2}}{16} .
$$

In this paper we combine the methods of [GLS00] and the method of Bogomolov instability to reproduce the result (1.2) in the plane case, and to derive a similar sufficient condition,

$$
\sum_{i=1}^{r} \gamma_{\alpha}\left(\mathcal{S}_{i}\right)<\gamma \cdot\left(D-K_{\Sigma}\right)^{2}
$$

for T-smoothness on other surfaces - involving a generalisation $\gamma_{\alpha}^{*}$ of the $\gamma_{1}^{*}$ invariant which is always bounded from above by the latter one.

Note that a series of irreducible plane curves of degree $d$ with $r$ singularities of type $A_{k}, k$ arbitrarily large, satisfying

$$
r \cdot k^{2}=\sum_{i=1}^{r} \tau^{*}\left(A_{k}\right)^{2}=9 d^{2}+\text { terms of lower order }
$$

constructed by Shustin (cf. Shu97]) shows that asymptotically we cannot expect to do essentially better in general. For a survey on other known results on $\Sigma=\mathbb{P}_{\mathbb{C}}^{2}$ we refer to [GLS00] and [GLS01], and for results on Severi varieties on other surfaces see [Tan80, GK89, GLS98, FM01, Fla01].

In this section we introduce the basic concepts and notations used throughout the paper, and we state several important known facts. Section 2 contains the main results and Section 3 their proofs.

1.1. General assumptions and notations. Throughout this article $\Sigma$ will denote a smooth projective surface over $\mathbb{C}$.

We will denote by $\operatorname{Div}(\Sigma)$ the group of divisors on $\Sigma$ and by $K_{\Sigma}$ its canonical divisor. If $D$ is any divisor on $\Sigma, \mathcal{O}_{\Sigma}(D)$ shall be the corresponding invertible sheaf and we will sometimes write $H^{\nu}(X, D)$ instead of $H^{\nu}\left(X, \mathcal{O}_{X}(D)\right)$. A curve $C \subset \Sigma$ will be an effective (non-zero) divisor, that is, a one-dimensional locally principal scheme, not necessarily reduced; however, an irreducible curve shall be reduced by definition. $|D|_{l}$ denotes the system of curves linearly equivalent to $D$. We will use the notation $\operatorname{Pic}(\Sigma)$ for the Picard group of $\Sigma$, that is, $\operatorname{Div}(\Sigma)$ modulo linear equivalence (denoted by $\sim_{l}$ ), and $\operatorname{NS}(\Sigma)$ for the Néron-Severi group, that is, $\operatorname{Div}(\Sigma)$ modulo algebraic equivalence (denoted by $\sim_{a}$ ). Given a reduced curve $C \subset \Sigma$ we will write $g(C)$ for its geometric genus.

Given any closed subscheme $X$ of a scheme $Y$, we denote by $\mathcal{J}_{X}=\mathcal{J}_{X / Y}$ the ideal sheaf of $X$ in $\mathcal{O}_{Y}$. If $X$ is zero-dimensional we denote by $\operatorname{deg}(X)=$ $\sum_{z \in Y} \operatorname{dim}_{\mathbb{C}}\left(\mathcal{O}_{Y, z} / \mathcal{J}_{X / Y, z}\right)$ its degree. If $X \subset \Sigma$ is a zero-dimensional scheme on $\Sigma$ and $D \in \operatorname{Div}(\Sigma)$, we denote by $\left|\mathcal{J}_{X / \Sigma}(D)\right|_{l}$ the linear system of curves $C$ in $|D|_{l}$ with $X \subset C$.

Given two curves $C$ and $D$ in $\Sigma$ and a point $z \in \Sigma$, and letting $f, g \in \mathcal{O}_{\Sigma, z}$ be local equations at $z$ of $C$ and $D$ respectively, we will then denote by $i(C, D ; z)=$ $i(f, g)=\operatorname{dim}_{\mathbb{C}}\left(\mathcal{O}_{\Sigma, z} /\langle f, g\rangle\right)$ the intersection multiplicity of $C$ and $D$ at $z$.

1.2. Singularity types. The germ $(C, z) \subset(\Sigma, z)$ of a reduced curve $C \subset \Sigma$ at a point $z \in \Sigma$ is called a plane curve singularity, and two plane curve singularities $(C, z)$ and $\left(C^{\prime}, z^{\prime}\right)$ are said to be topologically equivalent (respectively analytically 
equivalent) if there is a homeomorphism (respectively an analytical isomorphism) $\Phi:(\Sigma, z) \rightarrow\left(\Sigma, z^{\prime}\right)$ such that $\Phi(C)=C^{\prime}$. We call an equivalence class with respect to these equivalence relations a topological (respectively analytical) singularity type.

When dealing with numerical conditions for T-smoothness some topological (respectively analytical) invariants of the singularities play an important role. We gather some results on them here for the convenience of the reader.

Let $(C, z)$ be the germ at $z$ of a reduced curve $C \subset \Sigma$ and let $f \in R=\mathcal{O}_{\Sigma, z}$ be a representative of $(C, z)$ in local coordinates $x$ and $y$. For the analytical type of the singularity the Tjurina ideal

$$
I^{e a}(f)=\left\langle\frac{\partial f}{\partial x}, \frac{\partial f}{\partial y}, f\right\rangle
$$

plays a very important role, as does the equisingularity ideal

$$
I^{e s}(f)=\left\{g \in R \mid f+\varepsilon g \text { is equisingular over } \mathbb{C}[\varepsilon] /\left(\varepsilon^{2}\right)\right\} \supseteq I^{e a}(f)
$$

for the topological type. They give rise to the following invariants of the topological (respectively analytical) singularity type $\mathcal{S}$ of $(C, z)$.

(a) Analytical Invariants:

(1) $\tau(\mathcal{S})=\operatorname{dim}_{\mathbb{C}}\left(R / I^{e a}(f)\right)$ is the Tjurina number, i. e. the dimension of the base space of the semiuniversal deformation of $(C, z)$.

(2) $\tau_{c i}(\mathcal{S})=\max \left\{\operatorname{dim}_{\mathbb{C}}(R / I) \mid I^{e a}(f) \subseteq I\right.$ a complete intersection $\}$.

(3) $\gamma_{\alpha}^{e a}(\mathcal{S})=\max \left\{\gamma_{\alpha}(f ; I) \mid I^{e a}(f) \subseteq I\right.$ a complete intersection $\}$.

(b) Topological Invariants:

(1) $\tau^{e s}(\mathcal{S})=\operatorname{dim}_{\mathbb{C}}\left(R / I^{e s}(f)\right)$ is the codimension of the $\mu$-constant stratum in the semiuniversal deformation of $(C, z)$.

(2) $\tau_{c i}^{e s}(\mathcal{S})=\max \left\{\operatorname{dim}_{\mathbb{C}}(R / I) \mid I^{e s}(C, z) \subseteq I\right.$ a complete intersection $\}$.

(3) $\gamma_{\alpha}^{e s}(\mathcal{S})=\max \left\{\gamma_{\alpha}(f ; I) \mid I^{e s}(C, z) \subseteq I\right.$ a complete intersection $\}$.

Here, for an ideal $I$ containing $I^{e a}(f)$ and a rational number $0 \leq \alpha \leq 1$ we define $\gamma_{\alpha}(f ; I)=\max \left\{(1+\alpha)^{2} \cdot \operatorname{dim}_{\mathbb{C}}(R / I), \lambda_{\alpha}(f ; I, g) \mid g \in I, i(f, g) \leq 2 \cdot \operatorname{dim}_{\mathbb{C}}(R / I)\right\}$, where for $g \in I$

$$
\lambda_{\alpha}(f ; I, g)=\frac{\left(\alpha \cdot i(f, g)-(1-\alpha) \cdot \operatorname{dim}_{\mathbb{C}}(R / I)\right)^{2}}{i(f, g)-\operatorname{dim}_{\mathbb{C}}(R / I)} .
$$

Note that by Lemma $1.1 i(f, g)>\operatorname{dim}_{\mathbb{C}}(R / I)$ for all $g \in I$ and $\gamma_{\alpha}(f, g)$ is thus a well-defined positive rational number.

Throughout this article we will frequently treat topological and analytical singularities at the same time. Whenever we do so, we will write $\tau^{*}(\mathcal{S})$ for $\tau^{e s}(\mathcal{S})$, respectively for $\tau(\mathcal{S})$, and analogously we use the notation $\tau_{c i}^{*}(\mathcal{S})$ and $\gamma_{\alpha}^{*}(\mathcal{S})$. Analogously we will write $X^{*}(C)$ for the zero-dimensional schemes $X^{e s}(C)$, respectively for $X^{e a}(C)$ introduced in Subsection 1.3

One easily sees the following relations:

$$
(1+\alpha)^{2} \cdot \tau_{c i}^{*}(\mathcal{S}) \leq \gamma_{\alpha}^{*}(\mathcal{S}) \leq\left(\tau_{c i}^{*}(\mathcal{S})+\alpha\right)^{2} \leq\left(\tau^{*}(\mathcal{S})+\alpha\right)^{2} .
$$


In [LK03a] the $\gamma_{\alpha}^{*}$-invariant has been calculated for the simple singularities,

\begin{tabular}{|cc|c|}
\hline $\mathcal{S}$ & $\gamma_{\alpha}^{e a}(\mathcal{S})=\gamma_{\alpha}^{e s}(\mathcal{S})$ \\
\hline \hline$A_{k}$, & $k \geq 1$ & $(k+\alpha)^{2}$ \\
\hline$D_{k}, \quad 4 \leq k \leq 4+\sqrt{2} \cdot(2+\alpha)$ & $\frac{(k+2 \alpha)^{2}}{2}$ \\
\hline$D_{k}, \quad k \geq 4+\sqrt{2} \cdot(2+\alpha)$ & $(k-2+\alpha)^{2}$ \\
\hline$E_{k}, \quad c k=6,8$ & $\frac{(k+2 \alpha)^{2}}{2}$ \\
\hline
\end{tabular}

and for the topological singularity type $M_{m}$ of an ordinary $m$-fold point

$$
\gamma_{\alpha}^{e s}\left(M_{m}\right)=2 \cdot(m-1+\alpha)^{2} .
$$

Moreover, upper and lower bounds for the $\gamma_{0}^{e s}$-invariant and for the $\gamma_{1}^{e s}$-invariant of a topological singularity type given by a convenient semi-quasihomogeneous power series can be found there. They also show that

$$
\tau_{c i}^{e s}\left(M_{m}\right)=\left\{\begin{array}{cl}
\frac{(m+1)^{2}}{4}, & \text { if } m \geq 3 \text { odd } \\
\frac{m^{2}+2 m}{4}, & \text { if } m \geq 4 \text { even } \\
1, & \text { if } m=2 .
\end{array}\right.
$$

These results show in particular that the upper bound for $\gamma_{\alpha}^{*}(\mathcal{S})$ in (1.3) may be attained, while it may as well be far from the actual value.

The proof of the following lemma can be found in [Shu97], Lemma 4.1.

Lemma 1.1. Let $(C, z)$ be a reduced plane curve singularity given by $f \in \mathcal{O}_{\Sigma, z}$ and let $I \subseteq \mathfrak{m}_{\Sigma, z} \subset \mathcal{O}_{\Sigma, z}$ be an ideal containing the Tjurina ideal $I^{e a}(C, z)$. Then for any $g \in I$ we have

$$
\operatorname{dim}_{\mathbb{C}}\left(\mathcal{O}_{\Sigma, z} / I\right)<\operatorname{dim}_{\mathbb{C}}\left(\mathcal{O}_{\Sigma, z} /(f, g)\right)=i(f, g) .
$$

1.3. Singularity schemes. For a reduced curve $C \subset \Sigma$ we recall the definition of the zero-dimensional schemes $X^{e s}(C)$ and $X^{e a}(C)$ from GLS00. They are defined by the ideal sheaves $\mathcal{J}_{X^{e s}(C) / \Sigma}$ and $\mathcal{J}_{X^{e a}(C) / \Sigma}$ respectively, given by the stalks $\mathcal{J}_{X^{e s}(C) / \Sigma, z}=I^{e s}(f)$ and $\mathcal{J}_{X^{e a}(C) / \Sigma, z}=I^{\text {ea }}(f)$ respectively, where $f \in \mathcal{O}_{\Sigma, z}$ is a local equation of $C$ at $z$. We call $X^{e s}(C)$ the equisingularity scheme of $C$ and $X^{e a}(C)$ the equianalytical singularity scheme of $C$.

1.4. Equisingular families. Given a divisor $D \in \operatorname{Div}(\Sigma)$ and topological or analytical singularity types $\mathcal{S}_{1}, \ldots, \mathcal{S}_{r}$, we denote by $V=V_{|D|}\left(\mathcal{S}_{1}, \ldots, \mathcal{S}_{r}\right)$ the locally closed subspace of $|D|_{l}$ of reduced curves in the linear system $|D|_{l}$ having precisely $r$ singular points of types $\mathcal{S}_{1}, \ldots, \mathcal{S}_{r}$. By $V^{i r r}=V_{|D|}^{i r r}\left(\mathcal{S}_{1}, \ldots, \mathcal{S}_{r}\right)$ we denote the open subset of $V$ of irreducible curves. If a type $\mathcal{S}$ occurs $k>1$ times, we would rather write $k \mathcal{S}$ than $\mathcal{S}, \ldots ., \mathcal{S}$. We call these families of curves equisingular families of curves.

We say that $V$ is $T$-smooth at $C \in V$ if the germ $(V, C)$ is smooth of the (expected) dimension $\operatorname{dim}|D|_{l}-\operatorname{deg}\left(X^{*}(C)\right)$. By [Los98], Proposition 2.1 (see also GK89, GL96], GLS00]), T-smoothness of $V$ at $C$ follows from the vanishing of $H^{1}\left(\Sigma, \mathcal{J}_{X^{*}(C) / \Sigma}(C)\right)$, since the tangent space of $V$ at $C$ may be identified with $H^{0}\left(\Sigma, \mathcal{J}_{X^{*}(C) / \Sigma}(C)\right) / H^{0}\left(\Sigma, \mathcal{O}_{\Sigma}\right)$. 


\section{THE MAIN RESULTS}

In this section we give sufficient conditions for the T-smoothness of equisingular families of curves on certain surfaces with Picard number one, including the projective plane, general surfaces in $\mathbb{P}_{\mathbb{C}}^{3}$ and general K3-surfaces, on general products of curves, and on geometrically ruled surfaces. Since we do not have any general relation between the $\gamma$-invariants used in the conditions for our smoothness results and the invariants used in KT02 for the existence results, we are in general only able to say that the families produced here are T-smooth if they are non-empty. However, if you consider singularity types where the $\gamma$-invariant is known explicitely (see [LK03b]), you can check the conditions for non-emptiness in [KT02], and you will find that they are in general fulfilled as well.

\subsection{Surfaces with Picard number one.}

Theorem 2.1. Let $\Sigma$ be a surface such that $\mathrm{NS}(\Sigma)=L \cdot \mathbb{Z}$ with $L$ ample, let $D=d \cdot L \in \operatorname{Div}(\Sigma)$, let $\mathcal{S}_{1}, \ldots, \mathcal{S}_{r}$ be topological or analytical singularity types, and let $K_{\Sigma}=\kappa \cdot L$. Suppose that $d \geq \max \{\kappa+1,-\kappa\}$ and

$$
\sum_{i=1}^{r} \gamma_{\alpha}^{*}\left(\mathcal{S}_{i}\right)<\alpha \cdot\left(D-K_{\Sigma}\right)^{2}=\alpha \cdot(d-\kappa)^{2} \cdot L^{2} \quad \text { with } \alpha=\frac{1}{\max \{1,1+\kappa\}}
$$

Then either $V_{|D|}^{i r r}\left(\mathcal{S}_{1}, \ldots, \mathcal{S}_{r}\right)$ is empty or it is T-smooth.

Corollary 2.2. Let $d \geq 3$, let $H \subset \mathbb{P}_{\mathbb{C}}^{2}$ be a line, and let $\mathcal{S}_{1}, \ldots, \mathcal{S}_{r}$ be topological or analytical singularity types. Suppose that

$$
\sum_{i=1}^{r} \gamma_{1}^{*}\left(\mathcal{S}_{i}\right)<(d+3)^{2}
$$

Then either $V_{|d H|}^{i r r}\left(\mathcal{S}_{1}, \ldots, \mathcal{S}_{r}\right)$ is empty or T-smooth.

As soon as for one of the singularities we have $\gamma_{1}^{*}\left(\mathcal{S}_{i}\right)>4 \cdot \tau_{c i}^{*}\left(\mathcal{S}_{i}\right)$, e.g. simple singularities or ordinary multiple points which are not simple double points, then the strict inequality in (2.2) can be replaced by " $\leq$ ", which then is the same sufficient condition as in GLS01], Theorem 1 (see also (1.2)).

In particular, $V_{|d H|}^{i r r}\left(M_{m_{1}}, \ldots, M_{m_{r}}\right), m_{i} \geq 3$, is therefore T-smooth as soon as

$$
\sum_{i=1}^{r} 2 \cdot m_{i}^{2} \leq(d+3)^{2}
$$

Moreover, this condition has the right asymptotics, as the examples in GLS01. show. For further results in the plane case see Wah74, GK89, Lue87a, Lue87b. Shu87, Vas90, Shu91, Shu94 GL96, Shu96, Shu97, GLS98, Los98, GLS00, GLS01.

A smooth complete intersection surface with Picard number one satisfies the assumptions of Theorem 2.1. Thus by the Noether-Lefschetz Theorem (cf. GH85]) the result applies in particular to general surfaces in $\mathbb{P}_{\mathbb{C}}^{3}$. Moreover, if in Theorem 2.1 we have $\kappa>0$, i.e. $\alpha<1$, then the strict inequality in condition (2.1) may be replaced by " $\leq$ ", since in (3.9) the second inequality is strict, as is the second inequality in (3.10). 
Corollary 2.3. Let $\Sigma \subset \mathbb{P}_{\mathbb{C}}^{3}$ be a smooth hypersurface of degree $n \geq 5$, let $H \subset \Sigma$ be a hyperplane section, and suppose that the Picard number of $\Sigma$ is one. Let $d \geq n-3$ and let $\mathcal{S}_{1}, \ldots, \mathcal{S}_{r}$ be topological or analytical singularity types. Suppose that

$$
\sum_{i=1}^{r} \gamma_{\frac{1}{n-3}}^{*}\left(\mathcal{S}_{i}\right) \leq \frac{n}{n-3} \cdot(d-n+4)^{2}
$$

Then either $V_{|D|}^{i r r}\left(\mathcal{S}_{1}, \ldots, \mathcal{S}_{r}\right)$ is empty or it is T-smooth.

In particular, $V_{|d H|}^{i r r}\left(M_{m_{1}}, \ldots, M_{m_{r}}\right), m_{i} \geq 3$, is therefore T-smooth as soon as

$$
\sum_{i=1}^{r} 2 \cdot\left(m_{i}-\frac{n-4}{n-3}\right)^{2} \leq \frac{n}{n-3} \cdot(d-n+4)^{2},
$$

which is better than the conditions derived from [GLS97. The condition

$$
r \leq \frac{n \cdot(n-3)}{(n-2)^{2}} \cdot(d-n+4)^{2},
$$

which gives the T-smoothness of $V_{|d H|}\left(r A_{1}\right)$, is weaker than the condition provided in [CS97, but for $n=5$ it reads $r \leq \frac{10}{9} \cdot(d-1)^{2}$ and still comes close to the sharp bound $\frac{5}{4} \cdot(d-1)^{2}$ provided there for odd $d$.

A general K3-surface also has Picard number one..

Corollary 2.4. Let $\Sigma$ be a smooth K3-surface with $\mathrm{NS}(\Sigma)=L \cdot \mathbb{Z}, L$ ample, and set $n=L^{2}$. Let $d \geq 1$, and let $\mathcal{S}_{1}, \ldots, \mathcal{S}_{r}$ be topological or analytical singularity types. Suppose that

$$
\sum_{i=1}^{r} \gamma_{1}^{*}\left(\mathcal{S}_{i}\right)<d^{2} n
$$

Then either $V_{|d L|}^{i r r}\left(\mathcal{S}_{1}, \ldots, \mathcal{S}_{r}\right)$ is empty or it is T-smooth.

The best previously known condition for T-smoothness on K3-surfaces

$$
\sum_{i=1}^{r}\left(\tau_{c i}^{*}\left(\mathcal{S}_{i}\right)+1\right)^{2}<d^{2} n
$$

is thus completely replaced.

2.2. Products of curves. If $\Sigma=C_{1} \times C_{2}$ is the product of two smooth projective curves, then for a general choice of $C_{1}$ and $C_{2}$ the Néron-Severi group will be generated by two fibres of the canonical projections, by abuse of notation also denoted by $C_{1}$ and $C_{2}$. If both curves are elliptic, then "general" just means that the two curves are non-isogenous. (Cf. [Kei01] Appendix G.)

Theorem 2.5. Let $C_{1}$ and $C_{2}$ be two smooth projective curves of genera $g_{1}$ and $g_{2}$ with $g_{1} \geq g_{2}$, such that for $\Sigma=C_{1} \times C_{2}$ the Néron-Severi group is $\operatorname{NS}(\Sigma)=$ $C_{1} \mathbb{Z} \oplus C_{2} \mathbb{Z}$.

Let $D \in \operatorname{Div}(\Sigma)$ such that $D \sim_{a} a C_{1}+b C_{2}$ with $a \geq \max \left\{2-2 g_{2}, 2 g_{2}-1\right\}$ and $b \geq \max \left\{2-2 g_{1}, 2 g_{1}-1\right\}$, and let $\mathcal{S}_{1}, \ldots, \mathcal{S}_{r}$ be topological or analytical singularity types. Suppose that

$$
\sum_{i=1}^{r} \gamma_{0}^{*}\left(\mathcal{S}_{i}\right)<\gamma \cdot\left(D-K_{\Sigma}\right)^{2},
$$


where the constant $\gamma$ may be read off the following table with $A=\frac{a-2 g_{2}+2}{b-2 g_{1}+2}$ :

\begin{tabular}{|r|r|c|}
\hline$g_{1}$ & $g_{2}$ & $\gamma$ \\
\hline 0,1 & 0,1 & $\frac{1}{4}$ \\
\hline$\geq 2$ & 0,1 & $\min \left\{\frac{1}{4 g_{1}}, \frac{1}{4 \cdot\left(g_{1}-1\right) \cdot A}\right\}$ \\
\hline$\geq 2$ & $\geq 2$ & $\min \left\{\frac{1}{4 g_{1}+4 g_{2}-4}, \frac{A}{4 \cdot\left(g_{2}-1\right)}, \frac{1}{4 \cdot\left(g_{1}-1\right) \cdot A}\right\}$ \\
\hline
\end{tabular}

Then either $V_{|D|}^{\text {irr }}\left(\mathcal{S}_{1}, \ldots, \mathcal{S}_{r}\right)$ is empty or it is T-smooth.

In particular, on a product of non-isogenous elliptic curves for nodal curves we reproduce the previous sufficient condition

$$
r<\frac{a b}{2}
$$

for T-smoothness of $V_{\left|a C_{1}+b C_{2}\right|}^{i r r}\left(r A_{1}\right)$ from GLS97, while the previous general condition

$$
\frac{\left(m_{i}^{2}+2 m_{i}+5\right)^{2}}{32}<a b
$$

for T-smoothness of $V_{\left|a C_{1}+b C_{2}\right|}^{i r r}\left(M_{m_{1}}, \ldots, M_{m_{r}}\right), m_{i} \geq 3$, has been replaced by

$$
\sum_{i=1}^{r} 4 \cdot\left(m_{i}-1\right)^{2}<a b
$$

which is better from $m_{i}=7$ on.

Note that the coefficient $\gamma$ in Theorem 2.5 depends on the ratio of $a$ and $b$ unless both $g_{1}$ and $g_{2}$ are at most one. This means that in general an asymptotical behaviour can only be examined if the ratio remains unchanged.

2.3. Geometrically ruled surfaces. Let $\pi: \Sigma=\mathbb{P}_{\mathbb{C}}(\mathcal{E}) \rightarrow C$ be a geometrically ruled surface with normalised bundle $\mathcal{E}$ (in the sense of Har77 V.2.8.1). The Néron-Severi group of $\Sigma$ is $\mathrm{NS}(\Sigma)=C_{0} \mathbb{Z} \oplus F \mathbb{Z}$ with intersection matrix $\left(\begin{array}{cc}-e & 1 \\ 1 & 0\end{array}\right)$ where $F \cong \mathbb{P}_{\mathbb{C}}^{1}$ is a fibre of $\pi, C_{0}$ a section of $\pi$ with $\mathcal{O}_{\Sigma}\left(C_{0}\right) \cong \mathcal{O}_{\mathbb{P}(\mathcal{E})}(1), g=g(C)$ the genus of $C, \mathfrak{e}=\Lambda^{2} \mathcal{E}$ and $e=-\operatorname{deg}(\mathfrak{e}) \geq-g$. For the canonical divisor we have $K_{\Sigma} \sim_{a}-2 C_{0}+(2 g-2-e) \cdot F$.

Theorem 2.6. Let $\pi: \Sigma \rightarrow C$ be a geometrically ruled surface with $g=g(C)$. Let $D \in \operatorname{Div}(\Sigma)$ such that $D \sim_{a} a C_{0}+b F$ with $b>\max \left\{2 g-2+\frac{a e}{2}, 2-2 g+\frac{a e}{2}, a e\right\}$ and $a>2$, and let $\mathcal{S}_{1}, \ldots, \mathcal{S}_{r}$ be topological or analytical singularity types. Suppose that

$$
\sum_{i=1}^{r} \gamma_{0}^{*}\left(\mathcal{S}_{i}\right)<\gamma \cdot\left(D-K_{\Sigma}\right)^{2}
$$

where with $A=\frac{a+2}{b+2-2 g-\frac{a e}{2}}$ the constant $\gamma$ satisfies

$$
\gamma= \begin{cases}\frac{1}{4}, & \text { if } g \in\{0,1\}, \\ \min \left\{\frac{1}{4 g}, \frac{1}{4 \cdot(g-1) \cdot A}\right\}, & \text { if } g \geq 2 .\end{cases}
$$

Then either $V_{|D|}^{i r r}\left(\mathcal{S}_{1}, \ldots, \mathcal{S}_{r}\right)$ is empty or it is T-smooth. 
The results of [GLS97] only applied to eight Hirzebruch surfaces and a few classes of fibrations over elliptic curves, while our results apply to all geometrically ruled surfaces. Moreover, the results are in general better, e.g. for the Hirzebruch surface $\mathbb{P}_{\mathbb{C}}^{1} \times \mathbb{P}_{\mathbb{C}}^{1}$ already the previous sufficient condition for T-smoothness of families of curves with $r$ cusps and $b=3 a$ the condition

$$
9 r<2 a^{2}+8 a
$$

has been replaced by the slightly better condition

$$
8 r<3 a^{2}+8 a+4 .
$$

For ordinary multiple points the difference will become more significant. Even for families of nodal curves the new conditions would always be slightly better, but for those families T-smoothness is guaranteed anyway by Tan80].

Note that, as for products of curves, the coefficient $\gamma$ in Theorem 2.6 depends on the ratio of $a$ and $b$ unless $g$ is at most one.

\section{THE PROOFS}

The following lemma is the technical key to the above results. Using the method of Bogomolov unstable vector bundles, it gives us a "small" curve which passes through a "large" part of $X^{*}(C)$, provided that $h^{1}\left(\Sigma, \mathcal{J}_{X^{*}(C) / \Sigma}(D)\right) \neq 0$. We will then show that its existence contradicts (2.1), (2.3), or (2.4), respectively.

Lemma 3.1. Let $\Sigma$ be a smooth projective surface, and let $D \in \operatorname{Div}(\Sigma)$ and $X \subset \Sigma$ be a zero-dimensional scheme satisfying

(0) $D-K_{\Sigma}$ is big and nef, and $D+K_{\Sigma}$ is nef,

(1) $\exists C \in|D|_{l}$ irreducible: $X \subseteq X^{*}(C)$,

(2) $h^{1}\left(\Sigma, \mathcal{J}_{X / \Sigma}(D)\right)>0$, and

(3) $4 \cdot \operatorname{deg}\left(X_{0}\right)<\left(D-K_{\Sigma}\right)^{2}$ for all local complete intersection schemes $X_{0} \subseteq X$.

Then there exists a curve $\Delta \subset \Sigma$ and a zero-dimensional local complete intersection scheme $X_{0} \subseteq X \cap \Delta$ such that with the notation $\operatorname{supp}\left(X_{0}\right)=\left\{z_{1}, \ldots, z_{s}\right\}, X_{i}=$ $X_{0, z_{i}}$ and $\varepsilon_{i}=\min \left\{\operatorname{deg}\left(X_{i}\right), i\left(C, \Delta ; z_{i}\right)-\operatorname{deg}\left(X_{i}\right)\right\} \geq 1$ we have

(a) $D . \Delta \geq \operatorname{deg}\left(X_{0}\right)+\sum_{i=1}^{s} \varepsilon_{i}$

(b) $\operatorname{deg}\left(X_{0}\right) \geq\left(D-K_{\Sigma}-\Delta\right) \cdot \Delta$,

(c) $\left(D-K_{\Sigma}-2 \cdot \Delta\right)^{2}>0$, and

(d) $\left(D-K_{\Sigma}-2 \cdot \Delta\right) \cdot H>0$ for all $H \in \operatorname{Div}(\Sigma)$ ample.

Moreover, it follows that

$$
0 \leq \frac{1}{4} \cdot\left(D-K_{\Sigma}\right)^{2}-\operatorname{deg}\left(X_{0}\right) \leq\left(\frac{1}{2} \cdot\left(D-K_{\Sigma}\right)-\Delta\right)^{2} .
$$

Proof. Choose $X_{0} \subseteq X$ minimal such that still $h^{1}\left(\Sigma, \mathcal{J}_{X_{0} / \Sigma}(D)\right)>0$. By assumption (0) the divisor $D-K_{\Sigma}$ is big and nef, and thus $h^{1}\left(\Sigma, \mathcal{O}_{\Sigma}(D)\right)=0$ by the Kawamata-Viehweg Vanishing Theorem. Hence $X_{0}$ cannot be empty.

Due to the Grothendieck-Serre duality (cf. Har77] III.7.6) we have

$$
0 \neq H^{1}\left(\Sigma, \mathcal{J}_{X_{0} / \Sigma}(D)\right) \cong \operatorname{Ext}^{1}\left(\mathcal{J}_{X_{0} / \Sigma}\left(D-K_{\Sigma}\right), \mathcal{O}_{\Sigma}\right) .
$$

That is, there is an extension (cf. [Har77] Ex. III.6.1)

$$
0 \rightarrow \mathcal{O}_{\Sigma} \rightarrow E \rightarrow \mathcal{J}_{X_{0} / \Sigma}\left(D-K_{\Sigma}\right) \rightarrow 0
$$

\footnotetext{
${ }^{1}$ Since $X_{0} \subseteq X^{*}(C) \subseteq X^{e a}(C)$, Lemma 1.1 applies to the local ideals of $X_{0}$, that is, for the points $z \in \operatorname{supp}\left(X_{0}\right)$ we have $i(C, \Delta ; z) \geq \operatorname{deg}\left(X_{0}, z\right)+1$.
} 
The minimality of $X_{0}$ implies that $E$ is locally free and $X_{0}$ is a local complete intersection scheme (cf. [Laz97] Proposition 3.9). Moreover, we have (cf. [Laz97] Exercise 4.3)

$$
c_{1}(E)=D-K_{\Sigma} \quad \text { and } \quad c_{2}(E)=\operatorname{deg}\left(X_{0}\right) .
$$

By assumption (3) and (3.3) we have

$$
c_{1}(E)^{2}-4 \cdot c_{2}(E)=\left(D-K_{\Sigma}\right)^{2}-4 \cdot \operatorname{deg}\left(X_{0}\right)>0,
$$

and thus $E$ is Bogomolov unstable (cf. [Laz97] Theorem 4.2). This, however, implies that there exists a divisor $\Delta_{0} \in \operatorname{Div}(\Sigma)$ and a zero-dimensional scheme $Z \subset \Sigma$ such that

$$
0 \rightarrow \mathcal{O}_{\Sigma}\left(\Delta_{0}\right) \rightarrow E \rightarrow \mathcal{J}_{Z / \Sigma}\left(D-K_{\Sigma}-\Delta_{0}\right) \rightarrow 0
$$

is exact (cf. Laz97] Theorem 4.2), and such that

$$
\left(2 \Delta_{0}-D+K_{\Sigma}\right)^{2} \geq c_{1}(E)^{2}-4 \cdot c_{2}(E)>0
$$

and

$$
\left(2 \Delta_{0}-D+K_{\Sigma}\right) \cdot H>0 \quad \text { for all ample } \quad H \in \operatorname{Div}(\Sigma) .
$$

Tensoring (3.4) with $\mathcal{O}_{\Sigma}\left(-\Delta_{0}\right)$ leads to the following exact sequence:

$$
0 \rightarrow \mathcal{O}_{\Sigma} \rightarrow E\left(-\Delta_{0}\right) \rightarrow \mathcal{J}_{Z / \Sigma}\left(D-K_{\Sigma}-2 \Delta_{0}\right) \rightarrow 0,
$$

and we deduce $h^{0}\left(\Sigma, E\left(-\Delta_{0}\right)\right) \neq 0$.

Now tensoring (3.2) with $\mathcal{O}_{\Sigma}\left(-\Delta_{0}\right)$ leads to

$$
0 \rightarrow \mathcal{O}_{\Sigma}\left(-\Delta_{0}\right) \rightarrow E\left(-\Delta_{0}\right) \rightarrow \mathcal{J}_{X_{0} / \Sigma}\left(D-K_{\Sigma}-\Delta_{0}\right) \rightarrow 0 .
$$

Let $H$ be some ample divisor. By (3.6) and since $D-K_{\Sigma}$ is nef by (0):

$$
-\Delta_{0} \cdot H<-\frac{1}{2} \cdot\left(D-K_{\Sigma}\right) \cdot H \leq 0 .
$$

Hence $-\Delta_{0}$ cannot be effective, that is $H^{0}\left(\Sigma, \mathcal{O}_{\Sigma}\left(-\Delta_{0}\right)\right)=0$. But the long exact cohomology sequence of (3.8) then implies

$$
0 \neq H^{0}\left(\Sigma, E\left(-\Delta_{0}\right)\right) \hookrightarrow H^{0}\left(\Sigma, \mathcal{J}_{X_{0} / \Sigma}\left(D-K_{\Sigma}-\Delta_{0}\right)\right) .
$$

In particular we may choose a curve

$$
\Delta \in\left|\mathcal{J}_{X_{0} / \Sigma}\left(D-K_{\Sigma}-\Delta_{0}\right)\right|_{l} .
$$

Thus (c) and (d) follow from (3.5) and (3.6). It remains to show (a) and (b).

We note that $C \in|D|_{l}$ is irreducible and that $\Delta$ cannot contain $C$ as an irreducible component. Otherwise applying (3.6) with some ample divisor $H$ we would get the following contradiction, since $D+K_{\Sigma}$ is nef by $(0)$ :

$$
0 \leq(\Delta-C) \cdot H<-\frac{1}{2} \cdot\left(D+K_{\Sigma}\right) \cdot H \leq 0 .
$$

Since $X_{0} \subset C \cap \Delta$ the theorem of Bézout implies (a):

$$
\text { D. } \Delta=C . \Delta=\sum_{z \in C \cap \Delta} i(C, \Delta ; z) \geq \sum_{i=1}^{s}\left(\operatorname{deg}\left(X_{i}\right)+\varepsilon_{i}\right)=\operatorname{deg}\left(X_{0}\right)+\sum_{i=1}^{s} \varepsilon_{i} .
$$

Finally, by (3.3) and (3.4) we get (b):

$$
\operatorname{deg}\left(X_{0}\right)=c_{2}(E)=\Delta_{0} \cdot\left(D-K_{\Sigma}-\Delta_{0}\right)+\operatorname{deg}(Z) \geq\left(D-K_{\Sigma}-\Delta\right) . \Delta .
$$

Equation (3.1) is just a reformulation of (b). 
Using this result we can now prove the main theorems.

Proof of Theorem 2.1. Let $C \in V_{|D|}^{\text {irr }}\left(\mathcal{S}_{1}, \ldots, \mathcal{S}_{r}\right)$. It suffices to show that the cohomology group $h^{1}\left(\Sigma, \mathcal{J}_{X^{*}(C) / \Sigma}(D)\right)$ vanishes.

Suppose this is not the case. Since for $X_{0} \subseteq X^{*}(C)$ any local complete intersection scheme and $z \in \operatorname{supp}\left(X_{0}\right)$ we have

$$
4 \cdot \operatorname{deg}\left(X_{z}\right) \leq \frac{4}{(1+\alpha)^{2}} \cdot \gamma_{\alpha}^{*}(C, z) \leq \frac{1}{\alpha} \cdot \gamma_{\alpha}^{*}(C, z)
$$

Lemma 3.1 applies and there is a curve $\Delta \in|\delta \cdot L|_{l}$ and a local complete intersection scheme $X_{0} \subseteq X^{*}(C)$ satisfying the assumptions (a)-(d) there and (3.1). That is, fixing the notation $l=\sqrt{L^{2}}, \operatorname{supp}\left(X_{0}\right)=\left\{z_{1}, \ldots, z_{s}\right\}, X_{i}=X_{0, z_{i}}$ and $\varepsilon_{i}=$ $\min \left\{\operatorname{deg}\left(X_{i}\right), i\left(C, \Delta ; z_{i}\right)-\operatorname{deg}\left(X_{i}\right)\right\} \geq 1$, we have

(a) $d \cdot \delta \cdot l^{2} \geq \operatorname{deg}\left(X_{0}\right)+\sum_{i=1}^{s} \varepsilon_{i}$,

(b) $\operatorname{deg}\left(X_{0}\right) \geq(d-\kappa-\delta) \cdot \delta \cdot l^{2}$,

and

$\delta \cdot l \leq \frac{(d-\kappa) \cdot l}{2}-\sqrt{\frac{(d-\kappa)^{2} \cdot l^{2}}{4}-\operatorname{deg}\left(X_{0}\right)}=\frac{2 \cdot \operatorname{deg}\left(X_{0}\right)}{(d-\kappa) \cdot l+\sqrt{(d-\kappa)^{2} \cdot l^{2}-4 \cdot \operatorname{deg}\left(X_{0}\right)}}$.

But then together with (a) and (b) we deduce

$$
\sum_{i=1}^{s} \varepsilon_{i} \leq \delta \cdot(\delta+\kappa) \cdot l^{2} \leq \frac{1}{\alpha} \cdot\left(\frac{2 \cdot \operatorname{deg}\left(X_{0}\right)}{(d-\kappa) \cdot l+\sqrt{(d-\kappa)^{2} \cdot l^{2}-4 \cdot \operatorname{deg}\left(X_{0}\right)}}\right)^{2}
$$

Applying the Cauchy inequality this leads to

$$
\sum_{i=1}^{s} \frac{\operatorname{deg}\left(X_{i}\right)^{2}}{\varepsilon_{i}} \geq \frac{\operatorname{deg}\left(X_{0}\right)^{2}}{\sum_{i=1}^{s} \varepsilon_{i}} \geq \frac{\alpha \cdot(d-\kappa)^{2} \cdot l^{2}}{4} \cdot\left(1+\sqrt{1-\frac{4 \cdot \operatorname{deg}\left(X_{0}\right)}{(d-\kappa)^{2} \cdot l^{2}}}\right)^{2} .
$$

Setting

we thus have

$$
\beta=\frac{\sum_{i=1}^{s} \frac{\operatorname{deg}\left(X_{i}\right)^{2}}{\varepsilon_{i}}}{\alpha \cdot(d-\kappa)^{2} \cdot l^{2}}, \quad \gamma=\frac{\sum_{i=1}^{s} \frac{\operatorname{deg}\left(X_{i}\right)^{2}}{\varepsilon_{i}}}{\alpha \cdot \operatorname{deg}\left(X_{0}\right)}
$$

$$
\beta \geq \frac{1}{4} \cdot\left(1+\sqrt{1-\frac{4 \beta}{\gamma}}\right)^{2},
$$

and hence, $\beta \geq\left(\frac{\gamma}{\gamma+1}\right)^{2}$. But then, applying the Cauchy inequality once more, we find

$$
\begin{gathered}
\alpha \cdot(d-\kappa)^{2} \cdot l^{2}=\frac{\alpha \cdot \gamma}{\beta} \cdot \operatorname{deg}\left(X_{0}\right) \leq \alpha \cdot\left(\gamma+2+\frac{1}{\gamma}\right) \cdot \operatorname{deg}\left(X_{0}\right) \\
\leq \sum_{i=1}^{s}\left(\frac{\operatorname{deg}\left(X_{i}\right)^{2}}{\varepsilon_{i}}+2 \alpha \operatorname{deg}\left(X_{i}\right)+\alpha^{2} \varepsilon_{i}\right) \leq \sum_{i=1}^{r} \gamma_{\alpha}^{*}\left(\mathcal{S}_{i}\right),
\end{gathered}
$$

in contradiction to (2.1).

Proof of Theorem 2.5. Let $C \in V_{|D|}^{i r r}\left(\mathcal{S}_{1}, \ldots, \mathcal{S}_{r}\right)$. It suffices to show that the cohomology group $h^{1}\left(\Sigma, \mathcal{J}_{X^{*}(C) / \Sigma}(D)\right)$ vanishes.

Suppose this is not the case. Since for $X_{0} \subseteq X^{*}(C)$ any local complete intersection scheme and $z \in \operatorname{supp}(X)$ we have

$$
\operatorname{deg}\left(X_{z}\right) \leq \gamma_{0}^{*}(C, z)
$$


and since $\gamma \leq \frac{1}{4}$, Lemma 3.1 applies and there is curve $\Delta \sim_{a} \alpha \cdot C_{1}+\beta \cdot C_{2}$ and a local complete intersection scheme $X_{0} \subseteq X^{*}(C)$ satisfying the assumptions (a)-(d) there and (3.1). That is, fixing the notation $\operatorname{supp}\left(X_{0}\right)=\left\{z_{1}, \ldots, z_{s}\right\}, X_{i}=X_{0, z_{i}}$ and $\varepsilon_{i}=\min \left\{\operatorname{deg}\left(X_{i}\right), i\left(C, \Delta ; z_{i}\right)-\operatorname{deg}\left(X_{i}\right)\right\} \geq 1$, we have
(a) $a \beta+b \alpha \geq \operatorname{deg}\left(X_{0}\right)+\sum_{i=1}^{s} \varepsilon_{i}$,
(b) $\operatorname{deg}\left(X_{0}\right) \geq\left(a-2 g_{2}+2-\alpha\right) \cdot \beta+\left(b-2 g_{1}+2-\beta\right) \cdot \alpha$, and
(c) $0 \leq \alpha \leq \frac{a-2 g_{2}+2}{2}$ and $0 \leq \beta \leq \frac{b-2 g_{1}+2}{2}$.

The last inequalities follow from (d) in Lemma 3.1 replacing the ample divisor $H$ by the nef divisors $C_{2}$ respectively $C_{1}$.

From (b) and (c) we deduce

$$
\operatorname{deg}\left(X_{0}\right) \geq \frac{a-2 g_{2}+2}{2} \cdot \beta+\frac{b-2 g_{1}+2}{2} \cdot \alpha,
$$

and thus

$$
\operatorname{deg}\left(X_{0}\right)^{2} \geq 4 \cdot \frac{a-2 g_{2}+2}{2} \cdot \frac{b-2 g_{1}+2}{2} \cdot \alpha \cdot \beta=\frac{\left(D-K_{\Sigma}\right)^{2}}{2} \cdot \alpha \cdot \beta .
$$

Considering now (a) and (b) we get

$$
0<\sum_{i=1}^{s} \varepsilon_{i} \leq \Delta .\left(\Delta+K_{\Sigma}\right)=2 \alpha \beta+\left(2 g_{1}-2\right) \cdot \alpha+\left(2 g_{2}-2\right) \cdot \beta \leq \frac{\alpha \beta}{2 \gamma},
$$

where the last inequality holds only if $\alpha \neq 0 \neq \beta$. In particular, we see $\alpha \neq 0$ if $g_{2} \leq 1$ and $\beta \neq 0$ if $g_{1} \leq 1$. But this together with (3.11) gives

$$
\sum_{i=1}^{s} \varepsilon_{i} \leq \frac{\operatorname{deg}\left(X_{0}\right)^{2}}{\gamma \cdot\left(D-K_{\Sigma}\right)^{2}}
$$

If $\alpha=0$, then from (a) and (b) we deduce again

$$
0<\sum_{i=1}^{s} \varepsilon_{i} \leq\left(2 g_{2}-2\right) \cdot \beta \leq \frac{4 \cdot\left(g_{1}-1\right)}{A} \cdot \frac{\operatorname{deg}\left(X_{0}\right)^{2}}{\left(D-K_{\Sigma}\right)^{2}} \leq \frac{\operatorname{deg}\left(X_{0}\right)^{2}}{\gamma \cdot\left(D-K_{\Sigma}\right)^{2}},
$$

and similarly, if $\beta=0$,

$$
0<\sum_{i=1}^{s} \varepsilon_{i} \leq\left(2 g_{1}-2\right) \cdot \alpha \leq 4 \cdot\left(g_{1}-1\right) \cdot A \cdot \frac{\operatorname{deg}\left(X_{0}\right)^{2}}{\left(D-K_{\Sigma}\right)^{2}} \leq \frac{\operatorname{deg}\left(X_{0}\right)^{2}}{\gamma \cdot\left(D-K_{\Sigma}\right)^{2}} .
$$

Applying the Cauchy inequality, we finally get

$$
\gamma \cdot\left(D-K_{\Sigma}\right)^{2} \leq \frac{\operatorname{deg}\left(X_{0}\right)^{2}}{\sum_{i=1}^{s} \varepsilon_{i}} \leq \sum_{i=1}^{s} \frac{\operatorname{deg}\left(X_{i}\right)^{2}}{\varepsilon_{i}} \leq \sum_{i=1}^{r} \gamma_{0}^{*}\left(\mathcal{S}_{i}\right),
$$

in contradiction to Assumption (2.3).

Proof of Theorem 2.6. Let $C \in V_{|D|}^{\text {irr }}\left(\mathcal{S}_{1}, \ldots, \mathcal{S}_{r}\right)$. It suffices to show that the cohomology group $h^{1}\left(\Sigma, \mathcal{J}_{X^{*}(C) / \Sigma}(D)\right)$ vanishes.

Suppose this is not the case. Since for $X_{0} \subseteq X^{*}(C)$ any local complete intersection scheme and $z \in \operatorname{supp}(X)$ we have

$$
\operatorname{deg}\left(X_{z}\right) \leq \gamma_{0}^{*}(C, z),
$$

and since $\gamma \leq \frac{1}{4}$, Lemma 3.1 applies and there is curve $\Delta \sim_{a} \alpha \cdot C_{0}+\beta \cdot F$ and a local complete intersection scheme $X_{0} \subseteq X^{*}(C)$ satisfying the assumptions (a)-(d) there and (3.1). 
Remember that the Néron-Severi group of $\Sigma$ is generated by a section $C_{0}$ of $\pi$ and a fibre $F$ with intersection pairing given by $\left(\begin{array}{cc}-e & 1 \\ 1 & 0\end{array}\right)$. Then $K_{\Sigma} \sim_{a}-2 C_{0}+$ $(2 g-2-e) \cdot F$. Note that

$$
\alpha \geq 0 \quad \text { and } \quad \beta^{\prime}:=\beta-\frac{e}{2} \alpha \geq 0 .
$$

If we set $b^{\prime}=b-\frac{a e}{2}, \kappa_{1}=a+2$ and $\kappa_{2}=b+2-2 g-\frac{a e}{2}=b^{\prime}+2-2 g$, we get

$$
\left(D-K_{\Sigma}\right)^{2}=-e \cdot(a+2)^{2}+2 \cdot(a+2) \cdot(b+2+e-2 g)=2 \cdot \kappa_{1} \cdot \kappa_{2} .
$$

Now fixing the notation $\operatorname{supp}\left(X_{0}\right)=\left\{z_{1}, \ldots, z_{s}\right\}, X_{i}=X_{0, z_{i}}$, and finally $\varepsilon_{i}=$ $\min \left\{\operatorname{deg}\left(X_{i}\right), i\left(C, \Delta ; z_{i}\right)-\operatorname{deg}\left(X_{i}\right)\right\} \geq 1$, the conditions on $\Delta$ and $\operatorname{deg}\left(X_{0}\right)$ take the form

(a) $a \beta^{\prime}+b^{\prime} \alpha \geq \operatorname{deg}\left(X_{0}\right)+\sum_{i=1}^{s} \varepsilon_{i}$,

(b) $\operatorname{deg}\left(X_{0}\right) \geq \kappa_{1} \cdot \beta^{\prime}+\kappa_{2} \cdot \alpha-2 \alpha \beta^{\prime}$, and

(c) $0 \leq \alpha \leq \frac{\kappa_{1}}{2}$ and $0 \leq \beta^{\prime} \leq \frac{\kappa_{2}}{2}$.

The last inequalities follow from (d) in Lemma 3.1 replacing the ample divisor $H$ by the nef divisors $F$, respectively $C_{0}+\frac{e}{2} \cdot F$.

From (b) and (c) we deduce

$$
\operatorname{deg}\left(X_{0}\right) \geq \frac{\kappa_{1}}{2} \cdot \beta^{\prime}+\frac{\kappa_{2}}{2} \cdot \alpha,
$$

and thus, taking (3.12) into account,

$$
\operatorname{deg}\left(X_{0}\right)^{2} \geq 4 \cdot \frac{\kappa_{1}}{2} \cdot \frac{\kappa_{2}}{2} \cdot \alpha \cdot \beta^{\prime}=\frac{\left(D-K_{\Sigma}\right)^{2}}{2} \cdot \alpha \cdot \beta^{\prime} .
$$

Now considering (a) and (b) we get

$$
0<\sum_{i=1}^{s} \varepsilon_{i} \leq \Delta \cdot\left(\Delta+K_{\Sigma}\right)=2 \alpha \beta^{\prime}+(2 g-2) \cdot \alpha-2 \beta^{\prime} \leq \frac{\alpha \beta^{\prime}}{2 \gamma},
$$

where the last inequality holds if $\beta^{\prime} \neq 0$. We see, in particular, that $\beta^{\prime} \neq 0$ if $g \leq 1$. But this together with (3.13) gives for $\beta^{\prime} \neq 0$

$$
\sum_{i=1}^{s} \varepsilon_{i} \leq \frac{\operatorname{deg}\left(X_{0}\right)^{2}}{\gamma \cdot\left(D-K_{\Sigma}\right)^{2}}
$$

If $\beta^{\prime}=0$, then we deduce from (a) and (b)

$$
0<\sum_{i=1}^{s} \varepsilon_{i} \leq(2 g-2) \cdot \alpha \leq 4 \cdot(g-1) \cdot A \cdot \frac{\operatorname{deg}\left(X_{0}\right)^{2}}{\left(D-K_{\Sigma}\right)^{2}} \leq \frac{\operatorname{deg}\left(X_{0}\right)^{2}}{\gamma \cdot\left(D-K_{\Sigma}\right)^{2}} .
$$

Applying the Cauchy inequality, we finally get

$$
\gamma \cdot\left(D-K_{\Sigma}\right)^{2} \leq \frac{\operatorname{deg}\left(X_{0}\right)^{2}}{\sum_{i=1}^{s} \varepsilon_{i}} \leq \sum_{i=1}^{s} \frac{\operatorname{deg}\left(X_{i}\right)^{2}}{\varepsilon_{i}} \leq \sum_{i=1}^{r} \gamma_{0}^{*}\left(\mathcal{S}_{i}\right),
$$

in contradiction to assumption (2.4). 


\section{REFERENCES}

[CC99] Luca Chiantini and Ciro Ciliberto, On the Severi variety of surfaces in $\mathbb{P}_{\mathbb{C}}^{3}$, J. Algebraic Geom. 8 (1999), 67-83. MR.2000f:14082

[CS97] Luca Chiantini and Edoardo Sernesi, Nodal curves on surfaces of general type, Math. Ann. 307 (1997), 41-56. MR98b:14026

[Fla01] Flaminio Flamini, Moduli of nodal curves on smooth surfaces of general type, J. Algebraic Geom. 11 (2001), no. 4, 725-760. MR2003k:14028

[FM01] Flaminio Flamini and Carlo Madonna, Geometric linear normality for nodal curves on some projective surfaces, Bollettino UMI 4-B (2001), no. 8, 269-283. MR2001m:14046

[GH85] Philipp Griffiths and Joe Harris, On the Noether-Lefschetz theorem and some remarks on codimension-two cycles, Math. Ann. 271 (1985), no. 1, 109-205. MR87a:14030

[GK89] Gert-Martin Greuel and Ulrich Karras, Families of varieties with prescribed singularities, Comp. Math. 69 (1989), 83-110. MR90d:32037

[GL96] Gert-Martin Greuel and Christoph Lossen, Equianalytic and equisingular families of curves on surfaces, Manuscripta Math. 91 (1996), 323-342. MF98g:14023

[GLS97] Gert-Martin Greuel, Christoph Lossen, and Eugenii Shustin, New asymptotics in the geometry of equisingular families of curves, Internat. Math. Res. Notices 13 (1997), 595-611. MR.98g:14039

[GLS98] _ Geometry of families of nodal curves on the blown-up projective plane, Trans. Amer. Math. Soc. 350 (1998), 251-274. MF 98j:14034

[GLS00] — Castelnuovo function, zero-dimensional schemes, and singular plane curves, J. Algebraic Geom. 9 (2000), no. 4, 663-710. MR2001g:14045

[GLS01] - The variety of plane curves with ordinary singularities is not irreducible, Intern. Math. Res. Notes 11 (2001), 542-550. MR2002e:14042

[Har77] Robin Hartshorne, Algebraic geometry, Springer, 1977. MR57:3116

[Kei01] Thomas Keilen, Families of curves with prescribed singularities, Ph.D. thesis, Universität Kaiserslautern, 2001, http://www.mathematik.uni-kl.de/ keilen/download/Thesis/ thesis.ps.gz.

[KT02] Thomas Keilen and Ilya Tyomkin, Existence of curves with prescribed singularities, Trans. Amer. Math. Soc. 354 (2002), no. 5, 1837-1860, http://www.mathematik.uni-kl.

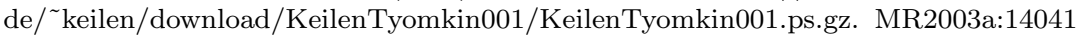

[Laz97] Robert Lazarsfeld, Lectures on linear series, Complex Algebraic Geometry (János Kollár, ed.), IAS/Park City Mathematics Series, no. 3, Amer. Math. Soc., 1997, pp. 161219. MR 98h:14008

[LK03a] Christoph Lossen and Thomas Keilen, The $\gamma_{\alpha}$-invariant for plane curve singularities, Preprint, 2003.

[LK03b] Christoph Lossen and Thomas Keilen, Injective analytic maps - a counterexample to the proof, Preprint, 2003.

[Los98] Christoph Lossen, The geometry of equisingular and equianalytic families of curves on a surface, Ph.d. thesis, FB Mathematik, Universität Kaiserslautern, Aug. 1998, http:// www.mathematik.uni-kl.de/ ${ }^{2}$ lossen/download/Lossen002/Lossen002.ps.gz.

[Lue87a] Ignacio Luengo, The $\mu$-constant stratum is not smooth, Inventiones Math. 90 (1987), 139-152. MR:88m:32021

[Lue87b] _ On the existence of complete families of plane curves, which are obstructed, J. LMS 36 (1987), 33-43. MR88f:14028

[Sev21] Francesco Severi, Vorlesungen über Algebraische Geometrie, Bibliotheca Mathematica Teubneriana, no. 32, Teubner, 1921.

[Shu87] Eugenii Shustin, Versal deformation in the space of plane curves of fixed degree, Funct. An. Appl. 21 (1987), 82-84. MR89a:14034

[Shu91] _ On manifolds of singular algebraic curves, Selecta Math. Sov. 10 (1991), 27-37.

[Shu94] Smoothness and irreducibility of varieties of algebraic curves with nodes and cusps, Bull. SMF 122 (1994), 235-253. MR95e:14020

[Shu96] - Smoothness and irreducibility of varieties of algebraic curves with ordinary singularities, Israel Math. Conf. Proc., no. 9, Amer. Math. Soc., 1996, pp. 393-416.

[Shu97] , Smoothness of equisingular families of plane algebraic curves, Math. Res. Not. 2 (1997), 67-82. MR 97j:14031 
[Tan80] Allen Tannenbaum, Families of algebraic curves with nodes, Compositio Math. 41 (1980), 107-126. MR82b:14017

[Tan82] , Families of curves with nodes on K3-surfaces, Math. Ann. 260 (1982), 239-253. MR84g:14025

[Vas90] Victor A. Vassiliev, Stable cohomology of complements to the discriminants of deformations of singularities of smooth functions, J. Sov. Math. 52 (1990), 3217-3230.

[Wah74] Jonathan M. Wahl, Deformations of plane curves with nodes and cusps, Amer. J. Math. 96 (1974), 529-577. MR52:8130

Fachbereich Mathematik, Universität Kaiserslautern, Erwin-Schrödinger-Strasse, D - 67663 Kaiserslautern, Germany

E-mail address: keilen@mathematik.uni-kl.de

URL: http://www.mathematik.uni-kl.de/ keilen 